\title{
Perioceutics in the management of Periodontal Disease
}

\author{
Rajeev Arunachalam*1, Vini Rajeev ',Vaishnavi Vedam¹, Sivadas Ganapathy', Jawahar Dhanavel ${ }^{2}$ \\ 'Lecturer, AIMST University, Semeling Bedong 08100, Kedah, MALAYSIA. \\ ${ }^{2}$ Tutor, AIMST University, Semeling Bedong 08100, Kedah, MALAYSIA.
}

\begin{abstract}
Periodontal disease is an immuno-inflammatory condition involving the tissues that surround and support the teeth. Till date back bone of periodontal treatment is still mechanical removal of plaque and calculus deposits from supra and sub gingival environment whereas complete elimination of these deleterious agents are quite unrealistic as the pocket depth increases. Intra pocket administration of drug via local drug delivery system have shown to achieve better clinical results when used as an adjunct to conventional non-surgical periodontal therapy, as periodontal pockets holds gingival crevicular fluid for the controlled release delivery of antimicrobials directly. This has steered the field of perioceutics which involves usage antimicrobial as well as host modulatory agents for the benefit of periodontium. Innovations in Perioceutics have led the researchers to have minimum usage of antibiotics as they develop resistance against microorganisms with side -effects. Presently focus is towards development of new Local Drug Delivery [LDD], host modulating agents, antibodies, biofilm which yield faster and safer results. In the article various locally delivered perioceutic agents are assessed with regard to their purpose, characteristics it possess, effectiveness as a monotherapy, incomparsion to scaling and root planning,
\end{abstract}

and ability to enhance conventional treatment. Furthermore, arguments related with local delivery are addressed

Key words: Antimicrobials, Bacteriostatic, Immune response, Host, Periodontal pocket.

Key message: Local drug delivery offers the clinician a new technique achieving and maintaining periodontal stability and thereby preventing further disease and subsequent problems like gum bleeding, mobility of tooth, pain and loss of tooth, periodontal abscess, tooth mobility and pain.

Correspondence:

Dr. Rajeev Arunachalam MDS,

Department of Periodontics, Faculty of Dentistry, AIMST University, Jalan Bedong Semeling, Kedah, Darul aman, MALAYSIA.

Phone: + 60164055204

E-mail: rajeevarun80@gmail.com

DOI: 10.5530/jyp.2017.9.3

\section{INTRODUCTION}

Infections affecting the supporting structures of the tooth are denoted as periodontal diseases. In the earliest stage the infection affects the gums/ gingiva and as disease progress, the supporting structures of the tooth which includes cementum, periodontal ligament and alveolar bone are affected resulting in loosening and prognosis of tooth. Periodontitis is most common disease entity affecting oral cavity next to dental caries. Basically periodontal diseases are bacteria induced infections where inflammation and destruction of the attachment apparatus occurs, often leading to tooth loss. ${ }^{1}$ Ignorance amongst population about oral care is the most prompting factor for increased incidence of periodontal disease. ${ }^{2}$ Periodontal diseases are multifactorial in nature which requires plaque for initiation followed by host microbial interactions. Actinobacillus actinomycetemcomitans, Porphyromonas gingivalis, Bacteroides forsythus and Treponema denticola are considered important causative organisms. ${ }^{3}$ Periodontal pockets are favorable area within the gums created by periodontopathic pathogens where access to routine cleaning are difficult leading to destruction of soft tissues supporting teeth and clinical attachment. Microorganisms reside in large numbers within the pocket and start releasing toxic substances harmful to host. In response to above the host as a part of immune response counteracts by releasing enzymes like MMPs (matrix metalloproteinase); inflammatory mediators like cytokines, prostanoids initially meant for defense and in later stages are responsible for destruction of periodontal tissue. ${ }^{4}$ Treatment strategy for the periodontal diseases must include the approaches that will target the microorganisms as well as modulate the destructive host response.
Heska Corporation first introduced the term perioceutic which is combination of terms periodontal and therapeutic which includes antimicrobial and host modulatory therapy in the management of periodontal disease along with mechanical debridement. ${ }^{4}$ Even though mechanical debridement removes plaque which contains microorganisms, it's impossible to completely eradicate all virulence factors; therefore antibacterial therapy is recommended as supplementary measure. When antimicrobials are administered systemically it exposes the body to large dose causing antibiotic resistance, adverse drug reaction and side effects, causing organ damage making it less patient compliant. ${ }^{3,5}$ Moreover less concentration of drug is achieved in Gingival Crevicular Fluid [GCF] when given systemically due to loss of drug during circulation to other parts of the body. ${ }^{6}$ Periodontal pockets form the primary source of infections with regard to periodontal infections; local drug delivery in the form of intra-pocket administration of drugs will prove to be more advantageous than systemic administration. ${ }^{3,7}$ Due to tissue invasive nature of some periodontal pathogens using mechanical therapy alone is ineffective. Therefore local administration of drugs can deliver higher concentrations directly into diseased sites reducing the microbial load. As a result advances in the local drug delivery [LDD] targeting periodontal pocket is a better approach in Perioceutics as high concentrations of drug at the target sites are attained with reduced systemic dosing, less applications, minor side effects, and high potential acceptability. ${ }^{8}$ The review article is an effort to examine various concepts of local drug delivery as relevant in periodontal diseases and to review the results of in vitro and in vivo studies demonstrating the chief role of these drugs when delivered direct into the periodontal pockets. 


\section{Rationale and Goal of Local Drug delivery in Periodontics}

Periodontal disease management has been directed at altering the periodontal surroundings to one, which is less favorable to the retention of bacterial plaque in and around gingival tissue.

Active phase of the disease can be changed intensely by decreasing the plaque levels.

Standard regimens to achieve this aim include

(1) Patient motivation and oral hygiene instructions

(2) Supra gingival scaling

(3) Correction of plaque retention local factors pertaining to restorative dentistry

(4) Subgingival scaling and Root planing

(5) Surgical elimination of pockets etc.

Principle behind LDD is that GCF contained within the pocket serves as a leaching medium for the discharge of a drug from the solid dosage form and for its dispersal throughout the pocket, making it a regular spot for treatment with local release delivery system.,10 The rational for the use of the local drug is to remove any residual infective /inflammatory element still harboring in the periodontal apparatus that are not reachable to mechanical removal by hand or motorized instruments. ${ }^{9,11}$ The most important goal is the prolonged obtainability of the drug in sufficient minimum inhibitory concentrations over a required period of time. Drugs used in LDD are summarized in Table $1.12,13$ The advantages and disadvantages are summarized in Table 2. ${ }^{13,14}$

Parameters include site of action, sufficient drug concentration available for ample duration of time, slower periodontal clearance and property of substantivity. ${ }^{12}$ Local drugs exert its action on bacteria present within periodontal pocket, soft tissue wall of pocket and on exposed cementum and radicular dentin but, the presence of subgingival calculus; anatomic anomalies, deep pockets and furcation lesions may pose physical difficulty in placing the drug at intended site which can be managed using intraoral irrigation devices. Substantivity pertaining to LDD refers to its ability to bind in the surrounding areas thereby creating a drug reservoir with slow release rate which can be attained by incorporating drug into various devices prior to placement in pocket. ${ }^{12}$ Indications and contraindications of LDD are depicted on Table $3 .{ }^{15}$

\section{Ideal requisites of locally delivered drug}

Basic and important factors that need to be fulfilled prior to usage of any antimicrobial recommended for periodontal disease management include: ${ }^{15}$

1. Drug should demonstrate in-vitro activity against microbes considered important for etiopathogenesis of the disease.

2. Within the subgingival environment the drug should be able to attain the desired dose concentration sufficient to eradicate microorganism.

3. That particular concentration of the drug should not have side effects and need to be specific for organisms causing periodontal disease.

\section{Dental applications of pharmacotherapeutic agents used in LDD:}

Tetracycline: It was first available LDD in fiber form. Made up of ethylene/ vinyl acetate copolymer fiber with diameter of $0.5 \mathrm{~mm}$, containing tetracycline $12.7 \mathrm{mg}$ per 9 inches. ${ }^{16}$ Antibacterial property of Tetracycline is due to a tetracyclic naphthacene carboxamide ring system with a dimethylamine group at carbon 4 (C4) in ring "A". Marketed under the name Actisite and have been approved by FDA. They are safe, inert, non resorbable copolymer with $25 \% \mathrm{w} / \mathrm{w}$ tetracycline HCI. It maintains persistent concentrations more than $1000 \mu \mathrm{g} / \mathrm{mL}$ for a period of 10 days. ${ }^{17}$ Other properties include collagenase inhibition, anti -bone resorption effect, anti-inflammatory actions and fibroblast attachment are of importance in management of periodontal diseases. ${ }^{18}$ Periodontal Plus $\mathrm{AB}$ is recently developed bioresorbable tetracycline fiber which degrades within 7 days and requires single appointment. Study done by Newman et al. on adjunctive tetracycline fiber therapy showed that fiber markedly improved the success of scaling and root planing in the treatment of localized recurrent periodontal cases and in supportive periodontal therapy. ${ }^{19}$ For subgingival delivery, tetracycline impregnated fibres of $0.5 \mathrm{~mm}$ in diameter made up of copolymer of ethylene vinyl acetate and tetracycline hydrochloride are used. It has been noted that a sustained high level of tetracycline averaging $1590 \mu \mathrm{g} / \mathrm{ml}$ delivering up to $25 \%$ of available tetracycline was observed following 10-days post placement in gingival crevicular fluid. ${ }^{20}$ Periodontal gel formulations containing tetracycline-serratiopeptidase has shown better results along with nonsurgical therapy. ${ }^{21}$ In summary, the studies reported that tetracycline fibers used as a monotherapy without non- surgical therapy were effective at decreasing probing depths, in gaining clinical attachment and reducing bacterial load. ${ }^{22,23}$

Doxycycline: Doxycycline is bacteriostatic synthetically prepared from oxytetracycline, acts by inhibiting bacterial protein synthesis. ${ }^{24}$ Major benefit of this drug over tetracycline is better patient acceptance as it given once a day. It can downregulate MMP enzyme, reducing collagenase activity that are capable of degrading a variety extracellular matrix. ${ }^{25}$ Controlled clinical trials have shown low dose of doxycycline was found to be effective in markedly decreasing the gingival inflammation and collagenase activity in the gingival crevicular fluid. ${ }^{25}$ Following 7 days post placement of doxycycline; levels were $6.0 \mu \mathrm{g} / \mathrm{mL}$, which exceeding the minimum inhibitory concentrations for periodontal microorganisms. After 28 days around 95\% of polymer is bio absorbed. ${ }^{26}$ Doxycline hyclate $10 \%$ as a local drug delivery was effective in reducing probing depth and gain in clinical attachment level..$^{27,28}$

- $\quad$ ATRIDOX is the only FDA approved $10 \%$ doxycycline in a gel system can reach to a level ranging $1,500-2000 \mu / \mathrm{ml}$ in $2 \mathrm{~h}$. ATRIDOX is supplied in two separate plastic syringes, one contains $42.5 \mathrm{mg}$ of doxycycline the other contains $450 \mathrm{mg}$ of the ATRIGEL Delivery System, which is a flowable polymeric preparation that is a mixture of $36.7 \%$ poly- DL-lactide dissolved in $63.3 \% \mathrm{~N}$-methyl2-pyrrolidone. The fillings of the two syringes are mixed and locally applied by placing it gently inside the periodontal pocket, which then literally flows to the bottom of the pocket and fills the gap between tooth and the gums. When applied, the gel hardens on contact with saliva to wax like consistency and is slowly released for 21 days. Studies have shown that bacteria including P. gingivalis and Fusobacterium nucleatum which are main causative agents, are vulnerable to doxycycline at concentrations of $6 \mathrm{mcg} / \mathrm{ml} .^{29}$

Sub gingival Minocycline: Bacteriostatic antibiotic available in three form: film, microspheres and ointment. ${ }^{30}$ Mechanism of action of Minocycline is by interfering with protein synthesis in the bacterial cell wall. ${ }^{31}$ Reduction in the bacterial number, complete eradication of spirochaetes for a period of 60 days with improvement in clinical parameters was noticed when minocycline was administered in a dosage of $200 \mathrm{mg}$ per day for 7 days $^{32}$ Repeated use of minocycline as an adjunct to the mechanical treatment of peri-implantitis showed positive results in probing depth reduction and was significantly different form the controls. ${ }^{33}$

- Film: Ethyl cellulose film contains 30\% of Minocycline may cause complete elimination of pathogenic flora after 14 days. ${ }^{34}$

- Microsphere: ARESTIN is a FDA approved sustained-release form of $2 \%$ minocycline microspheres. The each syringe contains 


\section{Table 1: Local Drug delivery in Periodontics}

Based on the application Personally applied (in patient home self-care)

Based on the application Professionally applied (in dental office)

Based on the duration of medicament release

Depending on degradability

Others
Non-sustained subgingival drug delivery

Traditional jet tips

Home oral irrigation

Home oral irrigation jet tips

Oral irrigation (water pick)

Soft cone rubber tips

Sustained sub-gingival drug delivery

Non-sustained sub gingival drug delivery

Professional pocket irrigation

Sustained subgingival drug delivery

Controlled release devices

Hollow fibres

Dialysis tubing

Strips

Films

Sustained release devices - Designed to provide drug delivery for less than 24 hours

Controlled release devices -drug release that at least exceeds 1 day - 3 days following application

Nondegradable devices (first generation)

Degradable devices (second generation)

Vehicles for supragingival drug delivery are mouth rinses, irrigation agents, chewing gums; and those used for subgingival drug delivery are controlled release system, monolithic fiber, hollow fiber acrylic strips and gels.

Table 2: Advantages and Disadvantages of local drug delivery

\begin{tabular}{|c|c|}
\hline Advantages & Disadvantages \\
\hline $\begin{array}{l}\text { Delivers drug in an optimal concentration } \\
\text { that can be maintained long enough for } \\
\text { the anticipated effect to be achieved } \\
\text { without causing any side effect. }\end{array}$ & Time consuming and labour -intensive \\
\hline Patient compliance better compared to systemic antibiotics & $\begin{array}{l}\text { Effect of local applied antimicrobials within the pockets will not be } \\
\text { effective in adjacent areas like tongue, tonsils, buccal mucosa, which } \\
\text { increases the chance of later infection }\end{array}$ \\
\hline $\begin{array}{l}\text { Alternative for management of individuals having high propensity } \\
\text { for gastro intestinal complications and for females with a propensity } \\
\text { for vaginal superinfections or other adverse reactions, from systemic } \\
\text { administration. }\end{array}$ & $\begin{array}{l}\text { Inaccessible areas in multirooted teeth like furcation cannot be dealt } \\
\text { with local drug delivery. }\end{array}$ \\
\hline \multicolumn{2}{|l|}{$\begin{array}{l}\text { Concentration of antimicrobial within subgingival environment can be } \\
\qquad 100 \text { fold higher compared to systemic. }\end{array}$} \\
\hline $\begin{array}{l}\text { Antibiotic resistance and superinfection are uncommon. } \\
\text { As a part home self-care certain forms of LDD can be applied by the } \\
\text { patient. }\end{array}$ & \\
\hline
\end{tabular}

Table3: Indications and Contraindications of LDD in periodontics

\begin{tabular}{|c|c|}
\hline Indications & Contraindications \\
\hline $\begin{array}{l}\text { In sites where instrumentation accessibility for scaling and root } \\
\text { planning difficult in case of deep periodontal pockets }\end{array}$ & Pregnant or Lactating patients if drug shows harmful effects \\
\hline In refractory periodontitis & $\begin{array}{l}\text { In aggressive form of periodontal disease where systemic antibiotics is } \\
\text { more effective }\end{array}$ \\
\hline $\begin{array}{l}\text { Failure to respond following repeated scaling and root planning in c } \\
\text { of localized pockets. }\end{array}$ & Patients who are allergic to components of LDD \\
\hline
\end{tabular}


microspheres of volume of equivalent to $4 \mathrm{mg}$, which is corresponding to $1 \mathrm{mg}$ of minocycline base., Prevotella intermedia, F. nucleatum, P. gingivalis A. Actinomycetemcomitans, and Eikenella corrodens are susceptible to Arestin.Once introduced subgingivally, the microspheres adhere to the walls of the pocket, where the polymer is hydrolyzed by the GCF causing water-filled channels to form inside the microspheres providing areas for the encapsulated antibiotic to be released. ${ }^{35}$ The minocycline diffuse from the microspheres over a 2-week period and end result is a level of $340 \mathrm{mcg} / \mathrm{ml}$ attained in the pocket. Having a advantage of ease of application and the drawback is it's a single-use product.

- Ointment: Dentomycin, Periocline are 2\% Minocycline gel reaches a concentration of $1300 \mu / \mathrm{ml}$ within I hour after application and reduced to $90 \mu / \mathrm{ml}$ after $7 \mathrm{hrs} .{ }^{36}$ It was shown that combination of minocycline ointment with non-surgical therapy like scaling and root planing showed significantly better results than debridement done alone.

Metronidazole: widely used as monotherapy and in combination with other antibiotics for the management for gingivitis, acute necrotizing ulcerative gingivitis, chronic periodontitis and Aggressive periodontitis

- $\quad E L Y Z O L$ is an oil based gel form contains 25\% metronidazole. Once applied the gel acquires more flow and fills pocket. Highly effective against obligate anaerobes, acts by inhibiting DNA synthesis. Studies reported for at least 8 hours concentrations of above $100 \mu / \mathrm{ml}$ were measurable in periodontal pocket and above $1 \mu / \mathrm{ml}$ after 36 hours $^{37}$ Effective in management of recurrent cases of chronic periodontitis as a supporting aid to conventional therapy. Gel is applied twice a week for a period of 14 day, where the dosage is dependent upon the number of teeth to be treated. For treating of pockets for around 6-8 teeth, $0.3 \mathrm{~g}$ of gel will be enough.

Subgingival Chlorhexidine: Commonly used as mouth rinses being highly recommended as an adjunct to tooth brushing and for the control of dental plaque. It reduces pellicle formation, altering the attachment of bacteria to tooth and affects the bacterial cell walls causing lysis. It acts by increasing the cellular membrane permeability of the bacteria resulting in coagulation of cytoplasmic macromolecules. Chlorhexidine exhibits high substantivity being cationic in nature. Available in the form of mouth rinses, Gels, varnishes and chip. Positively charged chlorhexidine molecule is enticed to the surface of the biologic membranes of bacterial and epithelial cells which is negatively charged. Mechanism of action includes adherence to bacterial cell wall, disrupting and entering the cell. Upon entry it disrupts the cytoplasm which drifts out of the damaged cell resulting in death of bacteria. ${ }^{10,38}$ Studies stated it to be self-retentive and gets degraded over the following 7 to 10 days. ${ }^{35,39}$ Around $40 \%$ of chlorhexidine is released over a period of $7-10$ days from its carrier with a mean concentration of $125 \mathrm{mcg} / \mathrm{ml}$ and $480 \mathrm{mcg} / \mathrm{ml}$ in 3 days. ${ }^{35,40}$ Following therapy with Periochip literature have shown suppression of the pocket flora for up to 11 weeks. ${ }^{41}$

Periochip: The Periochip was first launched in US dental market in 1998 weighing about $7.4 \mathrm{mg}$ and need to be refrigerated at 20-28 degree Celsius. Later in 2002 room temperature periochip was introduced with an advantage being simple to use and relatively easy to store with a longer shelf life 2 years. It consist of 10 chips per box. It's an orange brown colored biodegradable, rectangular chip form of $2.5 \mathrm{mg}$ of chlorhexidine gluconate that is released in a biphasic manner, initially releasing $40 \%$ within 24 hours and remaining over a time span of 7-10 days within the pocket. The polymer is made from $3.4 \mathrm{mg}$ of cross-linked hydrolyzed gelatin, $0.5 \mathrm{mg}$ of gelatin and $0.96 \mathrm{mg}$ of purified water, and contains $2.5 \mathrm{mg}$ chlorhexidine. ${ }^{25}$ Once the chip is placed within the confines of the pocket, its antiseptic property begin to kills microbes by slowly releasing the contents thereby shallowing of pocket occurs and inflammation get subsided. Hence promoting healing process and preventing further soft tissue or bone loss. Based on pharmacokinetic studies, release pattern of periochip into gingival sulcus after 10 days reflected that chlorhexidine levels in the gingival crevicular fluid were clinically effective for over one week period within the periodontal pockets with no signs of systemic absorption detected.

Periocol-C- Periocol chip is small orange-brown local drug delivery which is rectangular in shape; with one end rounded meant for ease of insertion into the periodontal pockets. Prepared by incorporating $2.5 \mathrm{mg}$ chlorhexidine from $20 \%$ chlorhexidine solution in type I biodegradable collagen membrane derived from fish sources. Within 24 hours Periocol releases approximately $40-45 \%$ chlorhexidine followed which in a rectilinear mode of dispersion for 7-8 days. This pattern of release may be described by initial burst effect due to diffusion of chlorhexidine from the local drug delivery system followed by release of the medication because of enzymatic degradation. Adjunctive usage of Periocol was regarded safe and delivered substantial improvement in both plaque development levels as shown by plaque indices and gingival bleeding levels as evidenced by scores of gingival bleeding index with the gain in clinical attachment level. ${ }^{42}$

- Chlosite -contains $1.5 \%$ chlorhexidine gel which is xanthan based preparation manufactured by Ghimas, Italy. Xanthan is saccharidic, biocompatible, naturally occurring polymer with a definite cross linkage pattern which controls the release of the drug. ${ }^{43}$ Formation of three -dimensional pseudoplastic reticulum by xanthan when contact in water, gives the ability to hold and maintain various substances in suspension. ${ }^{44}$ Sustained release of the drug is due to swelling -controlled erosional process which also goes through a progressive process of imbibition and following injection it is engrossed from the pocket within 10-30 days. It consist of blend of two preparations: Chlorhexidine dihydrochloride - 1\% which is slow releasing and Chlorhexidine digluconate - $0.5 \%$ with faster release. ${ }^{45}$ Following microorganisms are susceptible to chlorhexidine which includes P. gingivalis, F. nucleatum, P. intermedia, T. forsythia, C. rectus, H. aphrophilus. ${ }^{46}$ A concentration greater than $100 \mathrm{ug} / \mathrm{mL}$ of chlorhexidine digluconate is achieved on the first day which is then maintained for an average of 6-9 days reaching a level greater than the minimum inhibitory concentration for chlorhexidine $(0.10 \mathrm{ug} / \mathrm{mL})$. It's a gel form which degrades rapidly and is well tolerated and efficient in the management of periodontal pockets and peri implantitis. ${ }^{47}$

\section{Other local drug delivery systems-Future trends}

1. Clarithromycin gel: Subgingival delivery of $0.5 \%$ clarithromycin for management of chronic periodontitis in smoking patients along with conventional scaling and root planing have shown enhanced clinical outcome. Following 6 months all the clinical parameters such as probing pocket depth, clinical attachment level for clarithromycin group demonstrated marked improvement. The drug is yet tobe patented and under analysis. ${ }^{48}$

2. Herbal products- An abundant source of biologically active compounds are derived from natural products which have been the foundation for the advance of new lead substances for pharmaceuticals. Herbal medicines have better patient acceptance and tolerance. Various formulations like aloe vera, neem, tulsi, propolis, cocoa husk, pomegranate, cranberry etc. are being used widely these days. ${ }^{49,50}$

3. Colloidal drug carriers include micelles, emulsions, liposomes and nanoparticles.

4. PT-01 a sub gingival release delivery system containing Ofloxacin..$^{51}$ 


\section{CONCLUSION}

Currently due to lack of sufficient data it's difficult to substantiate one particular local drug delivery system is superior over the others. However local drug deliveries reduce the potential of developing antibiotic resistance and have greater range of success in refractory lesions which is localized with desired characteristics such as ease of placement, controlled release of drugs and resorbablity. Though the efficacy and practicality of local drug delivery have been confirmed by many clinical trials and have been approved by FDA for the management of periodontal disease, yet the risk/benefit ratio concerning to the usage of these drugs has yet to be established. Furthermore, continuous research in this field ensures and enables effective usage.

\section{CONFLICT OF INTEREST}

No conflict of interest are declared.

\section{ABBREVIATIONS USED}

LDD: Local Drug Delivery; MMPs: Matrix metalloproteinase; GCF: Gingival Crevicular Fluid.

\section{REFERENCES}

1. Genco J. Using antimicrobial agents to manage periodontal diseases. J Am Dent Assoc. 1991;122:31-8.

2. Yadav SK, Khan G, Mishra B. Advances in patents related to intra-pocket technology for the management of periodontitis. Recent Pat Drug Deliv Formula. 2015:9:129-45

3. Southard GL, Godowski KC. Sub gingival controlled release of antimicrobial agents in the treatment of periodontal disease. Int J Antimicrobial Agents. 1998;9:239-53.

4. Gulati M, Anand V, Govila V, Jain N. Host modulation therapy: An indispensable part of perioceutics. J Indian Soc Periodontal. 2014;18:282.

5. Walker CB. Selected antimicrobial agents: mechanisms of action, side effects and drug interactions. Periodontal 2000. 1996;10:12-28.

6. Goodson JM. Antimicrobial strategies for treatment of periodontal diseases. Periodontal 2000. 1994;5:142-68.

7. Schwach-Abdellaoui K, Vivien-Castioni N, Gurny R. Local delivery of antimicrobial agents for the treatment of periodontal diseases. European J Pharm Biopharm. 2000;50:83-99.

8. Chandrashekar KT, Sinha S. Effect of tetracycline hydrochloride on aspartate transaminase levels in chronic periodontitis. Hong Kong Dent. J 2011; 8: 8-24.

9. Shah R. Local Drug Delivery. Dental Dialogue. 2006;32(3):82-5.

10. Kornman KS. Controlled release local delivery-Antimicrobials in periodontics. Prospects for the future. J Periodontol.1993;64:782-91.

11. Kalsi R, Vandana KL, Prakash S. Effect of local drug delivery in chronic periodontitis patients: A meta-analysis. J Indian Soc Periodontol. 2011;15(4):304-9.

12. Greenstein G, Tonetti M. The role of controlled drug delivery for periodontitis. The Research, Science and Therapy Committee of the References American Academy of Periodontology. J Periodontl. 2000;71(1):125-40.

13. Rams TE, Slots J. Local delivery of antimicrobial agents in the periodontal pocket Periodontology 2000. 1996;10:139-59.

14. Dodwad V, Vaish S, Chhokra M, Mahajan A. Magic Bullet to treat Periodontitis: A targeted approach. J Pharm Biomed Sci. 2012;20(20):1-5.

15. Rose LF, Mealey BL, Genco RJ, CohenDW. Periodontics. China: Elsevier Mosby;2004.

16. Newman, Takei, Klokkevold, Carranza. Carranza's clinical periodontology 2010;pp 798-803, Chemotherapeutic agents, $10^{\text {th }}$ edition. 2010; Elsevier, Reed Elsevier India Private Limited.

17. Maurizio S, Tonetti. The topical use of antibiotics in periodontal pockets., in: N.P. Lang, T. Karring, J. Lindhe (Eds.), Proceedings of the Second European Workshop on Periodontology, Quintessence, London. 1997,109-32.

18. Soares PB, Menezes HH, Naves MM, Taga EM, Magalhaes D. Effect of absorbent tetracycline-loaded membrane used in the reduction of periodontal pockets: an in vivo study. Braz Dent J. 2009;20(5):414-8.

19. Newman MG, Kornman KS, Doherty FM. A 6-month multi-center evaluation of adjunctive tetracycline fiber therapy used in conjunction with scaling and root planing in maintenance patients: clinical results. J Periodontol. 1994;65:685-91.

20. Seymour RA, Heasman PA. Tetracyclines in the management of periodontal diseases. J Clin Periodontol. 1995;22:22-35.
21. Maheshwari M, Miglani G, Mali A, Paradkar A, Yamamura S, Kadam S. Development of Tetracycline-SerratiopeptidaseContaining Periodontal Gel: Formulation and Preliminary Clinical Study. AAPS Pharm Sci Tech. 2006;7(3):162-71.

22. Goodson JM, Hogan R, Dunham S. Clinical responses following periodontal treatment by local drug delivery. J Periodontol. 1985;56:81-7.

23. Sanz M, Serrano C, Garcia C, Echievarria C, O'Connor A. Clinical and microbiological efficacy of tetracycline fiber therapy in relapsing periodontal sites during supportive periodontal therapy. J Dent Res. 1997;76(Spec. Issue):153.

24. Perno M. Pharmacotherapy in Periodontal therapy. Access. 2001; Special supplemental issue: 1-11.

25. Preshow PM. Host response modulation in Periodontics. Periodontol. 2000:2008; 48:92-110.

26. Polson AM, Garrett S, Stoller NH, Bandt CL, Hanes PJ, Killoy WJ, et al. Multicenter comparative evaluation of subgingivally delivered sanguinarine and doxycycline in the treatment of Periodontitis. II. Clinical results. J Periodontol. 1997;68:119-26

27. Borden LC, Walker CB, Stone C, Mankodi S, Godowski KC, Southard H. Microbiota effects following sustained release subgingival delivery of doxycycline. J Dent Res. 1997;76(Spec.Issue):153.

28. Garrett S, Adams D, Bandt C, Beiswanger B, Bogle BC, Caton J, et al. Two multicenter clinical trials of subgingival doxycycline in the treatment of Periodontitis. J Dent Res. 1997:76(Spec. Issue):153

29. Garrett S, Johnson L, Drisko CH, Adams DF, Bandt C, Beiswanger B, et al. Two multi-center studies evaluating locally delivered doxycycline hyclate, placebo control, oral hygiene, and scaling and root planing in the treatment of periodontitis. J Periodontol. 1999;70:490-503.

30. Vandekerckhove BN, Quirynen M, Steenberghe V. The use of locally delivered minocycline in the treatment of chronic periodontitis- A review of the literature. J Clin Periodontol. 1998;25:964-8.

31. Tripathi KD. Essentials of medical pharmacology. 6t h ed. India: Jaypee Brothers; 2008.

32. Jain R, Mohamed F, Hemlatha M. Minocycline containing local drug delivery system in the management of chronic periodontitis: A randomized controlled trial. J Indian Soc Periodontol. 2012;16(2):179-83.

33. Renvert S, Lessem J, Dahlen G, Renvert H, Lindahl C. Mechanical and Repeated AntimicrobialTherapy Using a Local Drug DeliverySystemin theTreatment of Peri-Implantitis:A Randomized Clinical Trial. J Periodontol. 2008;79: 836-844.

34. Pragati S, Ashok S, Kuldeep S. Recent advances in periodontal drug delivery systems. Int J Drug Del. 2009;1:1-14.

35. Slots J, Rams TE. Antibiotics in periodontal therapy: advantages and disadvantages. J Clin Periodontol. 1990;17:479-93.

36. Van Steenebergh D, Bercy P, Kohl J. Subgingival Minocycline Hydrochloride ointment in moderate to severe chronic adult periodontitis a randomised double blind, vehicle controlled multicenter study. J Periodontol. 1993;64:637-44.

37. Chhina K, Bhatnagar R. Local drug delivery. Indian J Dent Sci. 2012;4:166-9.

38. De Graaff J, van Winkelhoff AJ, Goen RJ. The role of Actinobacillus actinomycetemcomitans in periodontal disease. Infection. 1989;17:269-71.

39. Stoller NH, Johnson LR, Trapnell S, Harrold CQ, Garrett S. The pharmacokinetic profile of a biodegradable controlled-release delivery system containing doxycycline compared to systemically delivered doxycycline in gingival crevicular fluid, saliva and serum. J Periodontol. 1998:69:1085-91.

40. Ciancio SG. Local delivery of chlorhexidine. Compend Contin Educ Dent. 1999;20:427-32

41. Paquette DW, Ryan ME, Wilder RS. Locally delivered antimicrobials: Clinical evidence and relevance. J Dent Hyg. 2008;82:10-5.

42. Kameswari K, Ambalavanan NT, RamakrishnaT, Saravana KR. Effectiveness of a controlled release chlorhexidine chip (PerioCol ${ }^{T M}-C G$ ) as an adjunctive to scaling and root planing when compared to scaling and root planing alone in the treatment of chronic periodontitis: A comparative study. J Indian Soc Periodontol. 2012;16(4): 553-7.

43. Maurstad G, Stokke BT. Metastable and stable states of xanthan polyelectrolyte complexes studies by atomic force microscopy. Biopolymers. 2004;74:199-213.

44. Zeng WM. Oral controlled release formulation for highly water soluble drugs: Drug-sodium alginate xanthan gum-zinc acetate matrix. Drug Dev Ind Pharm 2004;30:491-5.

45. Andreopoulos AG, Tarantili PA. Xanthan gum as a carrier for controlled release of drugs. J Biomater Appl. 2001;16:34-46.

46. Pratten J, Barnett P, Wilson M. Composition and susceptibility to chlorhexidine of multispecies biofilms of oral bacteria. Appl Environ Microbiol. 1998;64:35159.

47. NandaKumar P.K. Local Drug Delivery-Periocol in Periodontics. Trends Biomater Artif Organs. 2006;19(2);74-80.

48. Agarwal E, Pradeep AR, Bajaj P, Naik SB. Efficacy of local drug delivery of0.5\% clarithromycin gel as an adjunct to non-surgical periodontal therapy in the treatment of current smokers with chronic periodontitis: a randomized controlled clinical trial. J Periodontol. 2012;83(9):1155-63. 
49. Kukreja BJ, Dodwad V. Herbal Mouthwashes-A Gift of Nature: Int J Pharm Bio Sci. 2012;3:46-52. 47.

50. Bansal S, Rastogi S, Bajpai M. Mechanical, Chemical and Herbal Aspects of
Periodontitis: A Review. Int J Pharm Sci Res. 2012;3(5):1260-7.

51. Shah N. Local delivery of antimicrobial agents in periodontal therapy. J Indian Soc Periodontol. 1999;2:26-9.

Article History: Submission Date: 05-08-16; Revision Date: 13-09-16; Accepted Date:15-09-16.

Cite this article: Arunachalam R, Rajeev V, Vedam V, Ganapathy S, Dhanavel J Perioceutics in the management of Periodontal Disease. J Young Pharm. 2017;9(1):8-13. 